\title{
Regional Phase Correction of Inversion-Recovery MR Images
}

\author{
Joseph A. Borrello, Thomas L. Chenevert, And Alex M. Aisen \\ Department of Radiology, University of Michigan Hospitals, 1500 E. Medical Center Dr., \\ Ann Arbor, Michigan 48109-0030
}

Received November 29, 1988; revised June 16, 1989

\begin{abstract}
Many MR imaging systems are limited in their ability to successfully display inversionrecovery images. The reason is that part of the contrast is encoded as phase differences between pixels, whereas in the more commonly used spin-echo pulse sequence all the information is contained in the pixel magnitude. Inversion-recovery images are often displayed in magnitude form, resulting in loss of potentially useful phase information contained in the data. Before this phase information can be used, phase errors which result from scanner imperfections must be removed. While most of the necessary correction can be accomplished using data obtained by scanning a uniform phantom, this approach has several disadvantages. An alternative method by which phase errors can be readily removed without phantom data is described. This method has been applied to images of the head, knee, and liver with good results. It is concluded that this technique is useful for producing phase corrected inversion-recovery MR images. (C) 1990 Academic Press, Inc.
\end{abstract}

\section{INTRODUCTION}

Inversion-recovery (IR) images acquired using many commercially available MR scanners are produced using only the magnitude of the spatial-domain data and not the phase information. As a result ambiguity is introduced into the image, and pixels with different T1's may have the same intensity (1). This can negate much of the advantage of IR images for optimizing $\mathrm{T} 1$ contrast.

To avoid this ambiguity, a method of phase-sensitive image reconstruction is necessary. Unfortunately, factors other than T1 can cause phase shifts in the image--for example pulse sequence timing errors, gradient eddy currents, magnet inhomogeneity, and motion/flow effects. These effects can, in part, be compensated for by imaging a uniform phantom and using phase information from the phantom image to correct the phase of the patient image. This technique does not correct phase errors which are unique to the patient, however. Another method has been described for correcting the images using multiple IR acquisitions (at least four) with different inversion times (2). Also previously described are techniques which correct phase based on assumptions about the nature of the phase errors $(3,4)$, other than the assumption that an abrupt $180^{\circ}$ transition represents a $\mathrm{T} 1$ interface and not a phase error. A method which avoids some of the problems with these techniques is described here. This approach corrects the phase directly, without using a phantom, without using multiple IR acquisitions, and without making assumptions about the phase errors. 


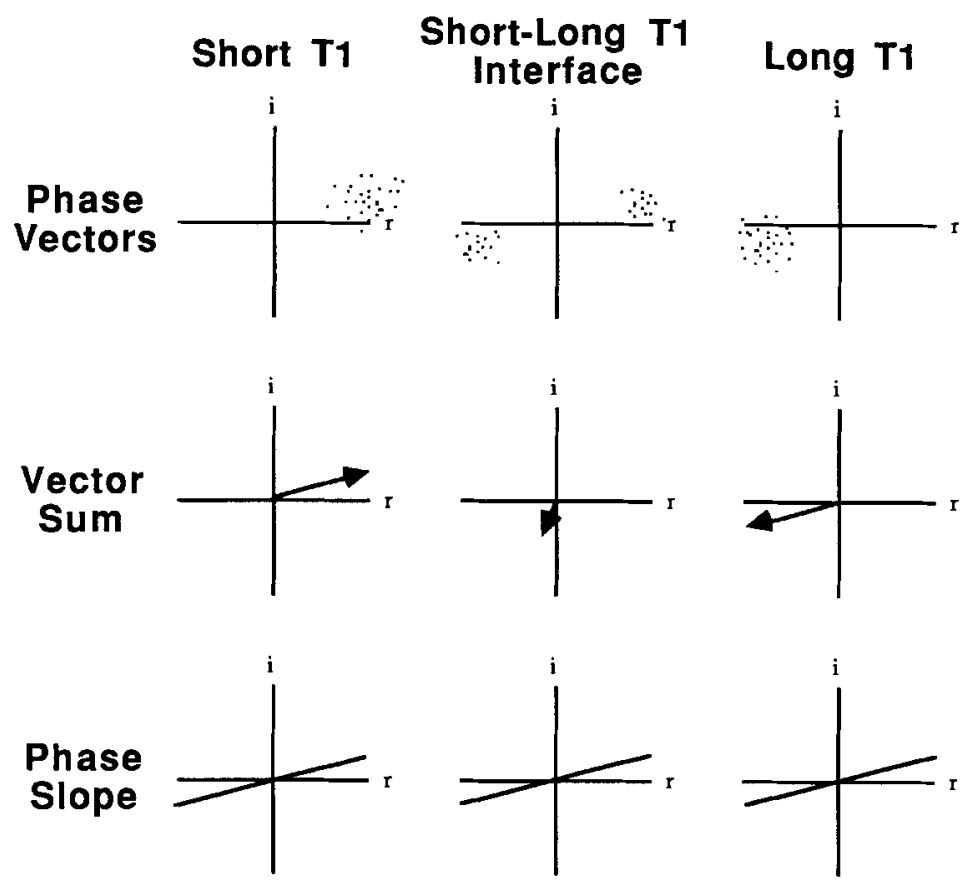

FIG. 1. Diagrammatic representation of phase-slope. Axes represent the real and imaginary axes of the complex plane.

\section{METHODS}

Scanning was performed using a $0.35 \mathrm{~T}$ superconductive whole-body imager. All sequences used a repetition time (TR) of $1500 \mathrm{~ms}$ and an echo time (TE) of $30 \mathrm{~ms}$. Inversion time (TI) was either 100 or $200 \mathrm{~ms}$. Spatial resolution was $1.7 \times 1.7 \mathrm{~mm}$, and slice thickness was $10 \mathrm{~mm}$. There were a total of 25 brain images of three patients, 24 knee images of two patients, and 24 liver images of two patients.

Phase-corrected IR images were obtained using a regional phase correction algorithm, run on a VAX 11-750 computer with a standard floating-point coprocessor (Digital Equipment Corporation, Marlboro, MA). The goal is to remove the gradual phase changes due to machine imperfections while preserving the abrupt $180^{\circ}$ phase changes due to interfaces between tissues with differences in T1. This algorithm begins correcting phase near the center of the image and gradually moves outward, using information from pixels which have been phase corrected to correct the phase of adjacent pixels. The method used by the phase correction algorithm involves the computation of a map of the gradual phase shifts due to machine imperfections. This map is used to correct the phase of the original image.

The first step in obtaining this map is to perform a $5 \times 5$-pixel kernal low-pass phase filtering operation on the complex image, thereby calculating an "average" value for each pixel. However, this average value is not a vector sum of the neighboring complex vectors but instead is what shall be referred to as "phase-slope." The meaning of this term is illustrated in Fig. 1. A small area of the image may contain 


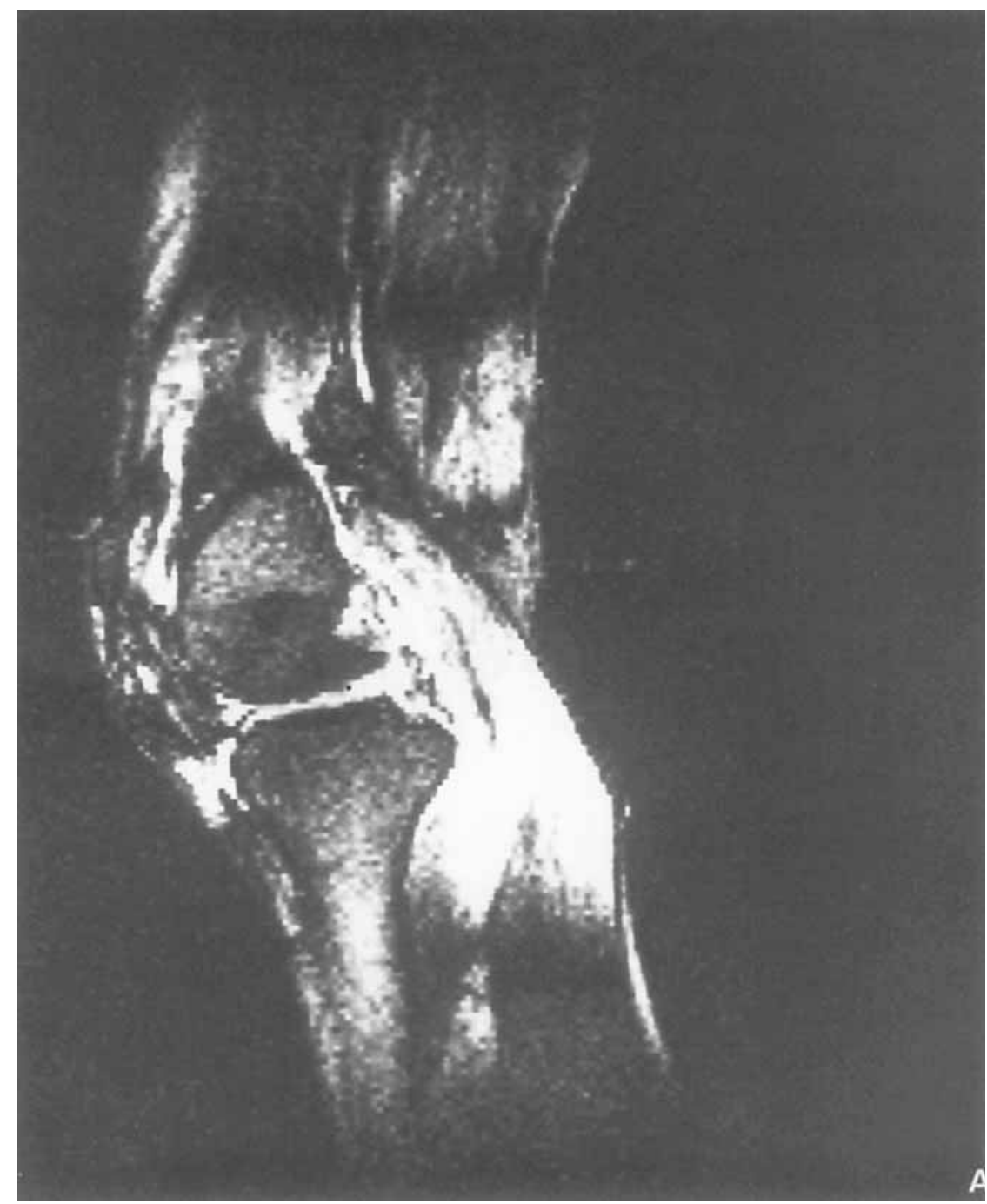

FIG. 2. Steps in the process of regional phase correction. (A) Modulus image. (B) Phase image prior to phase correction. (C) Phase-slope of B. (D) Partial phase unwrapping of C. In all phase images the grayscale is set such that a phase of $-179^{\circ}$ is black and a phase of $+180^{\circ}$ is white.

pixels of one of two opposite phases depending on the T1 of tissues at that location. It may also contain pixels of both opposite phases if the area is at an interface of two tissues of sufficiently different $\mathrm{T} 1$ 's. These three possibilities are represented in the three columns of Fig. 1. The top row of Fig. 1 shows collections of complex valued pixels. The middle row shows the results after performing a vector sum on the phase in each of these three situations. Areas of short T1 will have vector sums $180^{\circ}$ out of phase with areas of long $\mathrm{T} 1$, and areas along the interface will have unpredictable vector sums because of cancellation of opposing phases. Instead of using a vector 


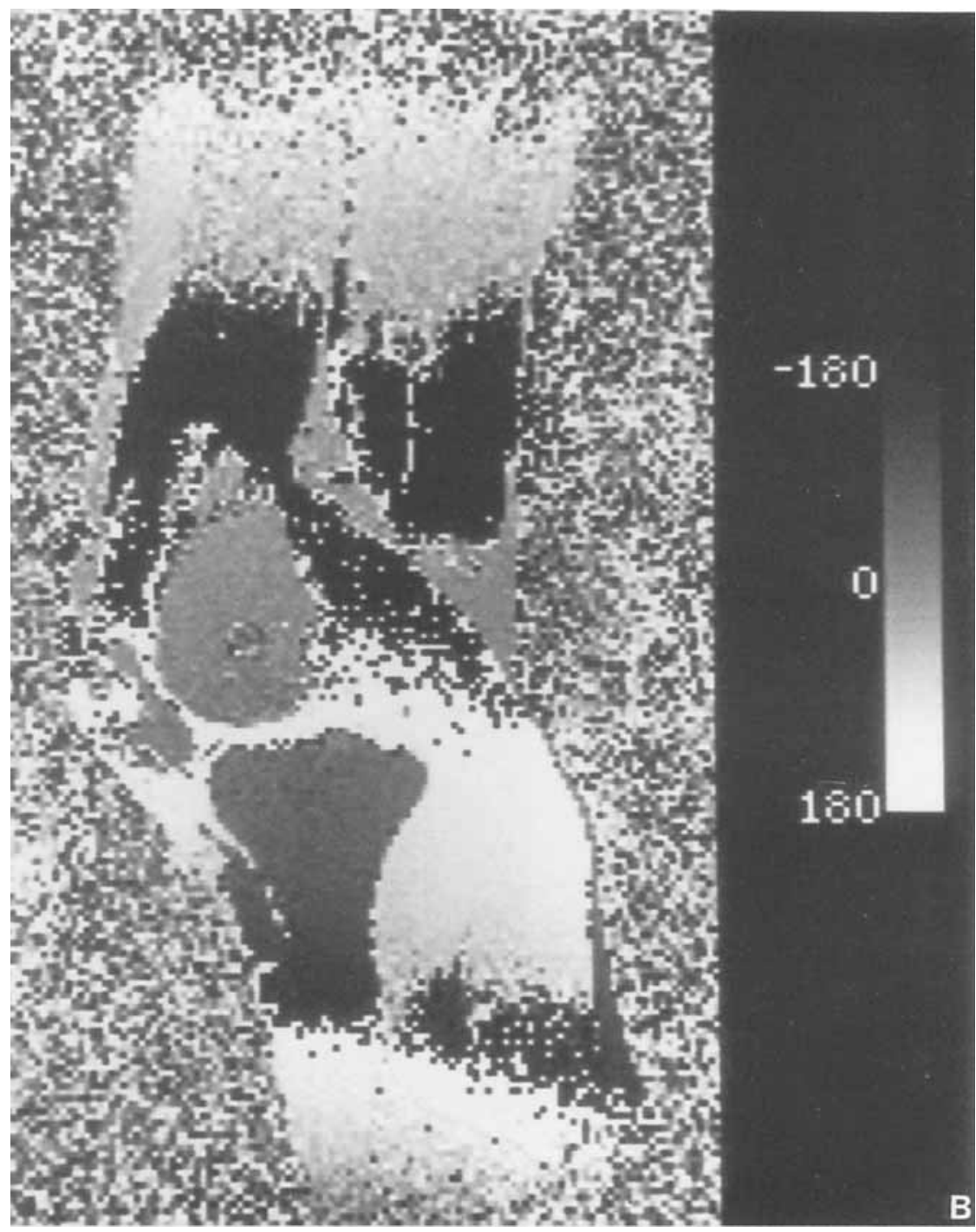

FIG. 2-Continued

sum, the regional phase correction algorithm uses a least-squares routine to fit a line to the neighborhood complex pixels as shown in the bottom row of Fig. 1. This line is constrained to pass through the origin of the complex plane, therefore the only parameter that the fit routine needs to determine is the slope of the line, which we refer to as the phase-slope. As can be seen in Fig. 1, areas with similar non-T1 phase effects will have similar values of phase-slope whether they are short T1, long T1, or an interface. Therefore plotting the phase-slope will remove abrupt $180^{\circ}$ phase transitions but preserve gradual phase shifts.

Figure 2 shows an example of this process performed on a sagittal IR image of a knee. Figure $2 \mathrm{~A}$ is a modulus image, and Fig. $2 \mathrm{~B}$ is a phase image produced from the 


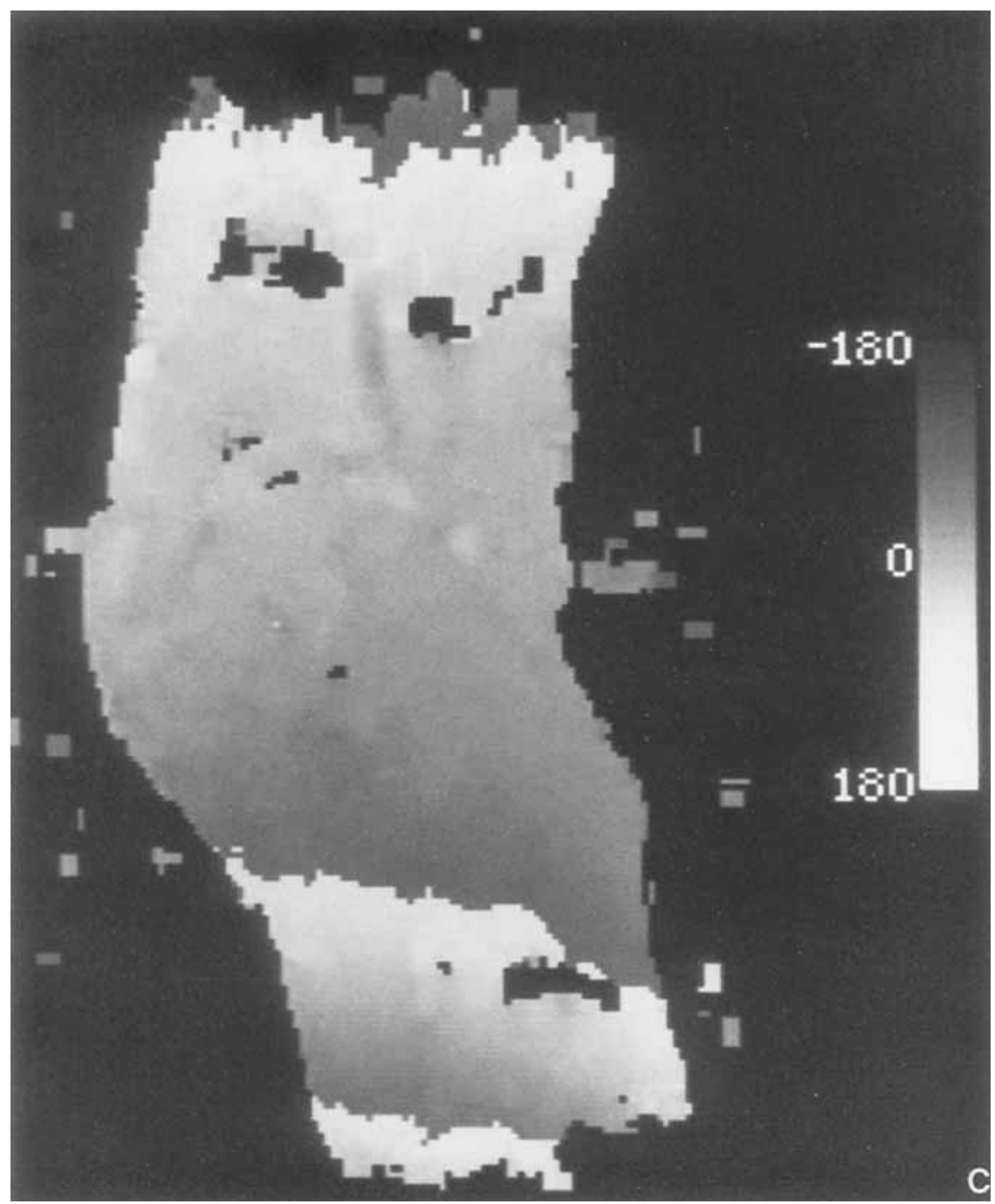

FIG. 2-Continued

complex spatial-domain data prior to phase correction. Figure $2 \mathrm{C}$ shows the result of calculating the phase-slope of this image. The slopes are represented as their arctangents and therefore all have values between $-90^{\circ}$ and $+90^{\circ}$. It is apparent that the $180^{\circ}$ phase difference between short $\mathrm{T} 1$ (such as bone marrow) and long T1 (such as muscle) has been removed in the phase-slope plot.

As can also be seen in Fig. $2 \mathrm{C}$ the phase-slope map is ambiguous regarding the phase of each individual pixel. This is due to the fact that a line of a given slope can represent either of two antiparallel phase vectors. In other words one cycle of the original phase image maps onto the phase-slope image as two cycles. To produce a true map of the non-T1 phase shifts one must first assign a phase value between $-90^{\circ}$ 


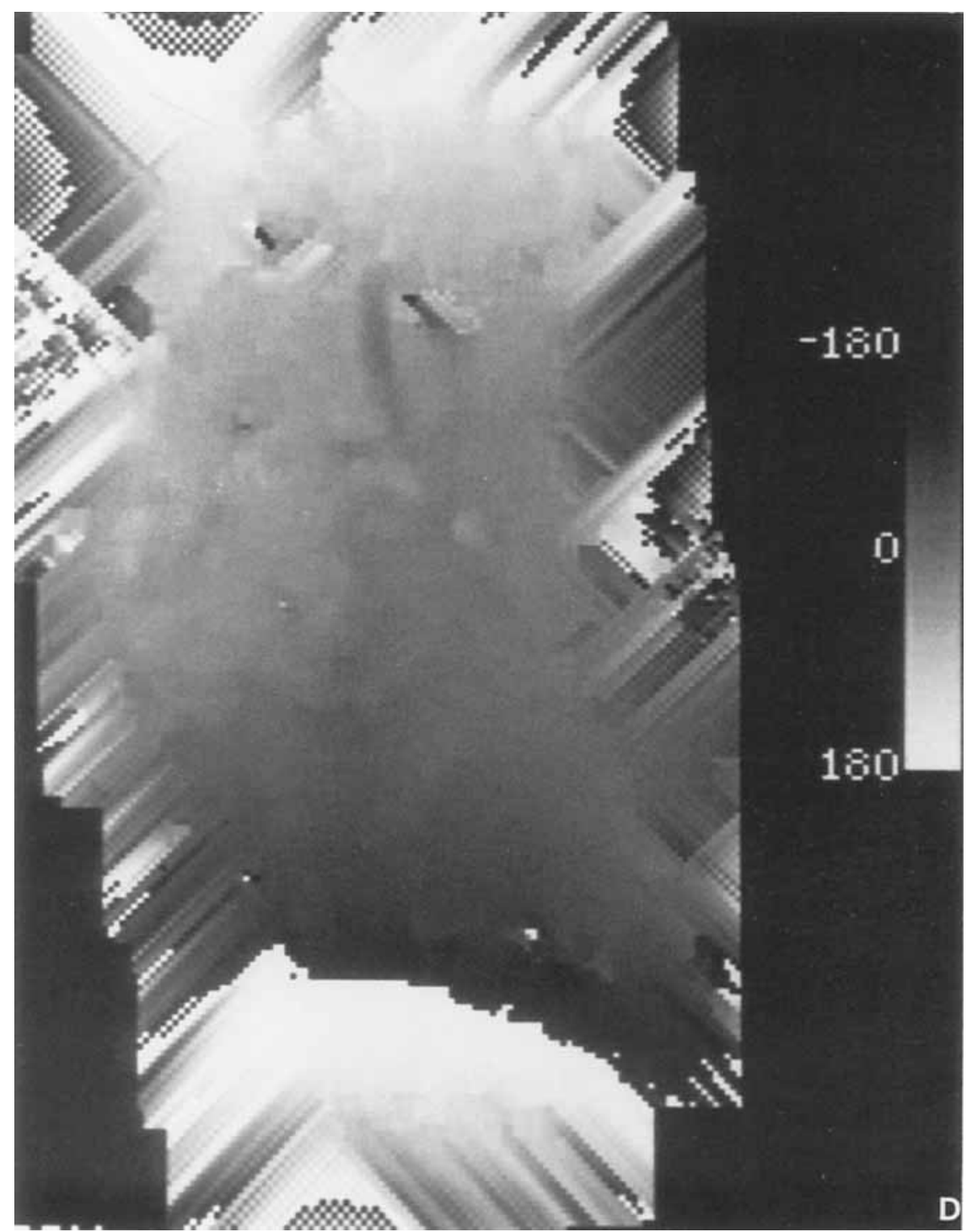

FIG. 2-Continued

and $+90^{\circ}$ to each phase-slope (i.e., calculate the two-quadrant arctangent), and then perform a partial phase unwrapping on the phase-slope map. The unwrapping is done as follows. The algorithm begins unwrapping at an area of the image near the center which has pixel magnitudes above a prespecified noise threshold. As one moves through the phase-slope map in a direction of increasing slope, eventually a slope with an arctangent of $+90^{\circ}$ will be reached. The next pixel will have a slope with an arctangent of, perhaps, $-89^{\circ}$. The unwrapped phase for this pixel will be assigned $+91^{\circ}$, and as the phase-slopes of the subsequent pixels approach $0^{\circ}$ the unwrapped phase will approach $+180^{\circ}$. At this point the next pixel will be assigned an 


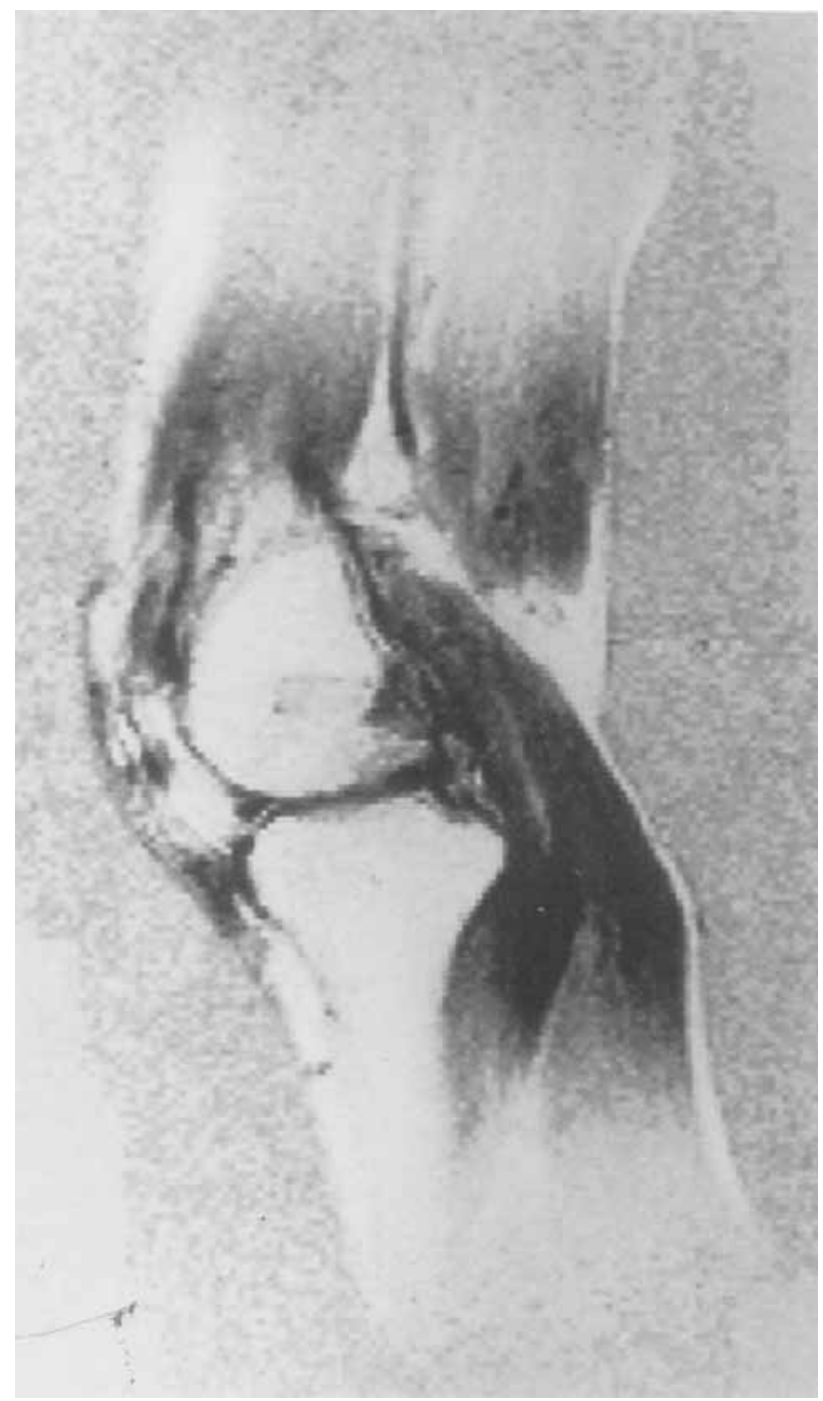

FIG. 3. Final corrected image of the knee of Fig. 2. IR 1500/30/100.

unwrapped phase of $-179^{\circ}$. This partial unwrapping removes the abrupt $-90^{\circ}$ to $+90^{\circ}$ transitions. The result of this operation is a map of the machine imperfection phase effects (Fig. 2D).

In the final step of regional phase correction the phase of each pixel of the original image is compared to the corresponding pixel of the calculated phase map. Depending on whether these two vectors are closer to being parallel or antiparallel (dot product $>$ or < zero) the magnitude of each pixel image is assigned a plus sign or a minus sign, respectively. This assignment of a sign to the magnitude is equivalent to rotating the corrected image vectors onto the nearest part of the real axis. This process will 


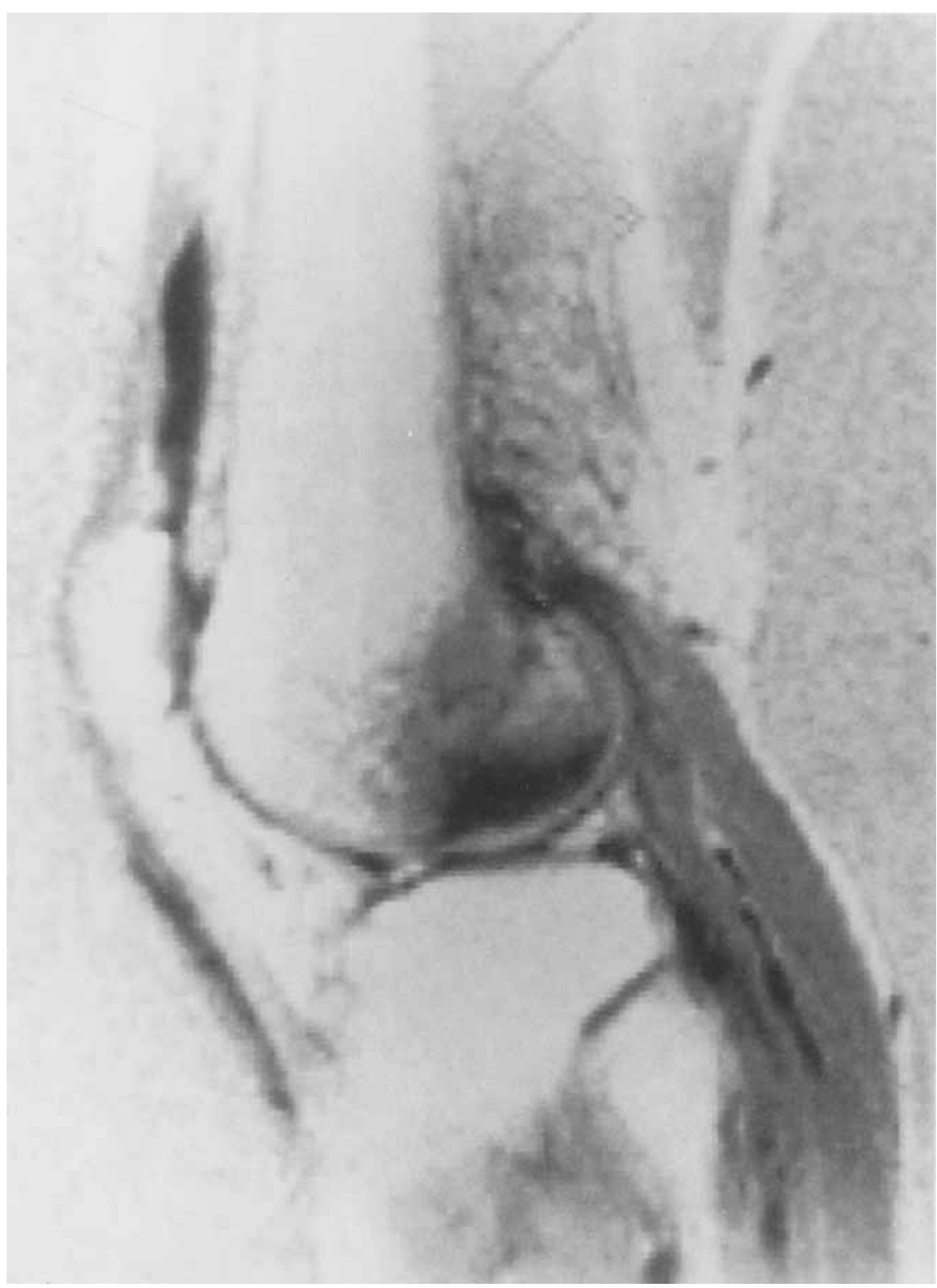

FIG. 4. Sagittal image of a knee with a probable infarct of the lateral condyle of the fermur. IR 1500/30/ 100. A joint effusion is also present.

remove any residual phase errors which are less than $90^{\circ}$. Thus as long as the phase map values are correct to within $\pm 90^{\circ}$, the appearance of the final image will be correct. When these signed values are displayed such that negative numbers correspond to the black side of the gray scale and positive numbers to the white, a true inversionrecovery image results (Fig. 3). 


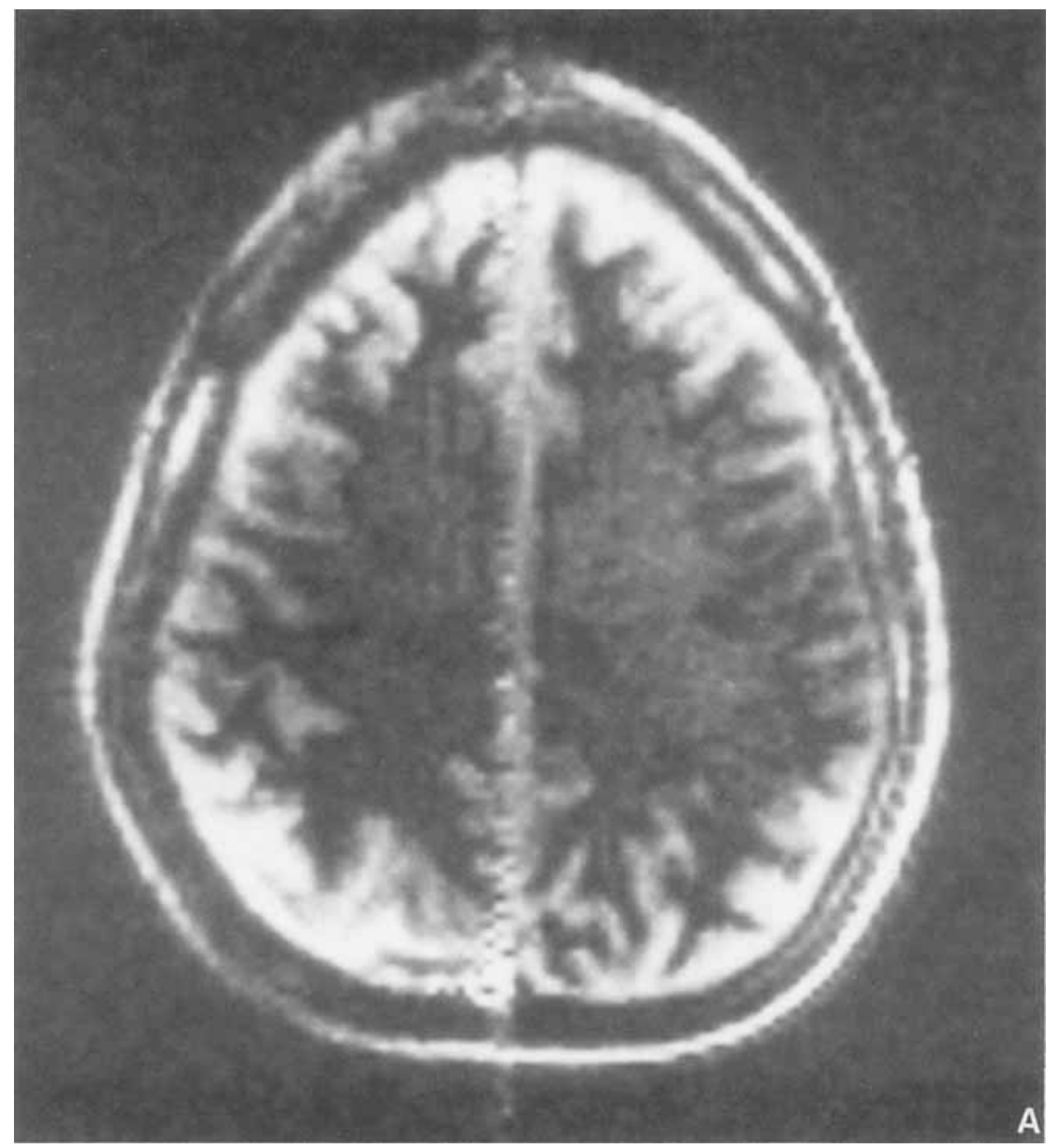

FIG. 5. Axial image of the brain of a patient with multiple sclerosis. IR 1500/30/200. (A) Modulus image. (B) Phase corrected image. Note the improved visualization of the lesions in the white matter on the phase corrected image.

As one would expect, pixels which represent noise or ghosting artifact have phases which are not interdependent with adjacent pixels, and the regional phase correction process becomes random in such areas. However, since correction of pixels relies on the results of correction of adjacent pixels, areas of noise can introduce errors which are propagated beyond the point at which they originally occur. To prevent this, such areas are avoided on the first pass of phase correction, thereby using only true signal to perform phase correction. Following this first pass, the noisy areas are filled in. Such areas are recognized by two criteria: defining a magnitude threshold below which pixels are avoided, and defining a threshold of phase coherence (i.e., the degree to which phase vectors in a small region are parallel or antiparallel to each other, rather than oriented randomly) below which pixels are also avoided. The threshold of magnitude is selected empirically for each set of images by visual inspection of the 


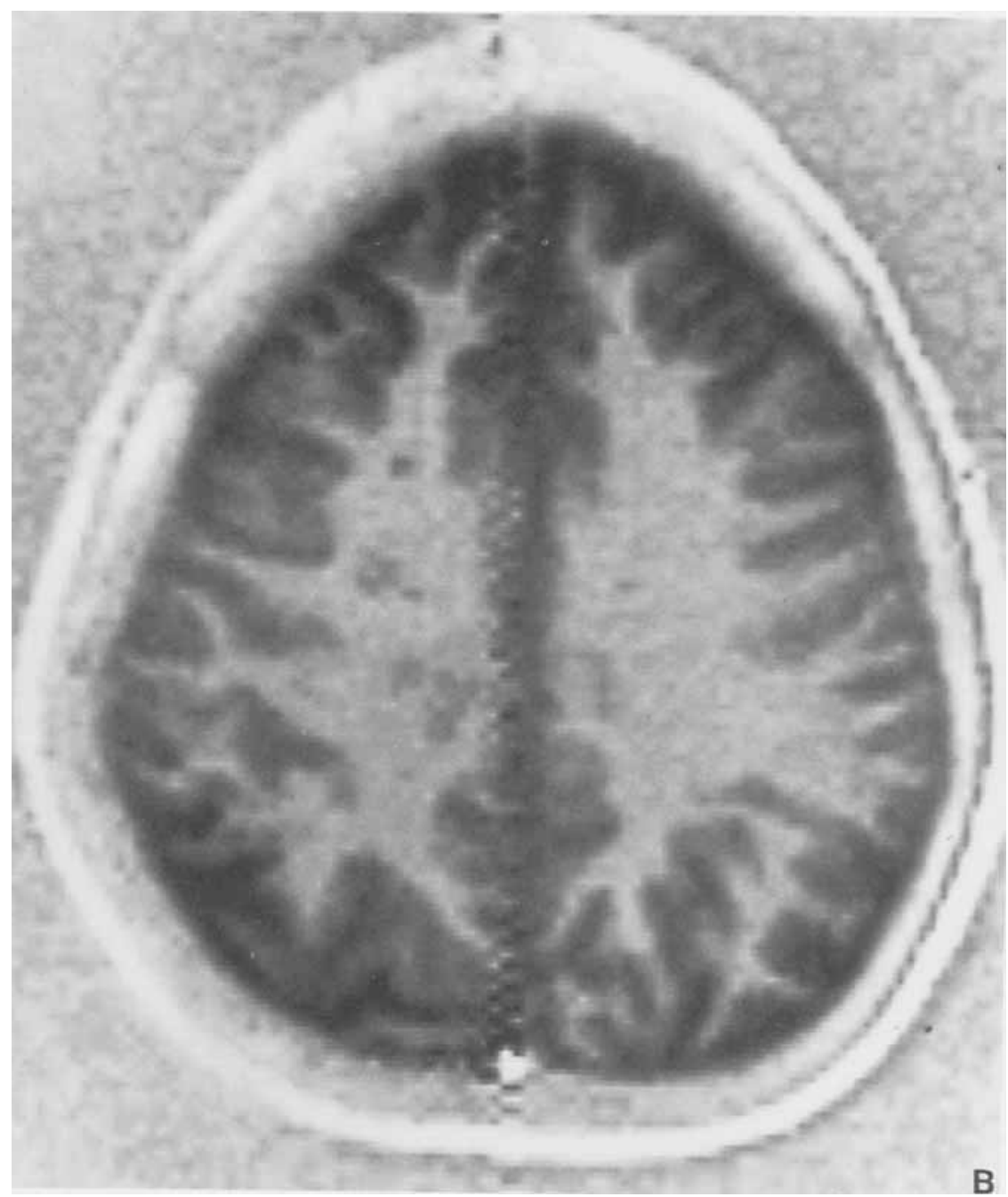

FIG. 5-Continued

center slice. The threshold of coherence is selected empirically on the basis of values which have worked with previous patients. The images are inspected after phase correction, and any images which contain phase errors are corrected with a different coherence threshold. Usually only about $\frac{1}{4}$ of images need to be redone, and usually no more than once or twice. Isolated areas surrounded by low signal are linked with adjacent areas using a linear extrapolation method.

\section{RESULTS}

Examples of images obtained with this technique are shown in Figs. 4-6. In general, images with good signal-to-noise ratios and little motion artifact (such as images of the 


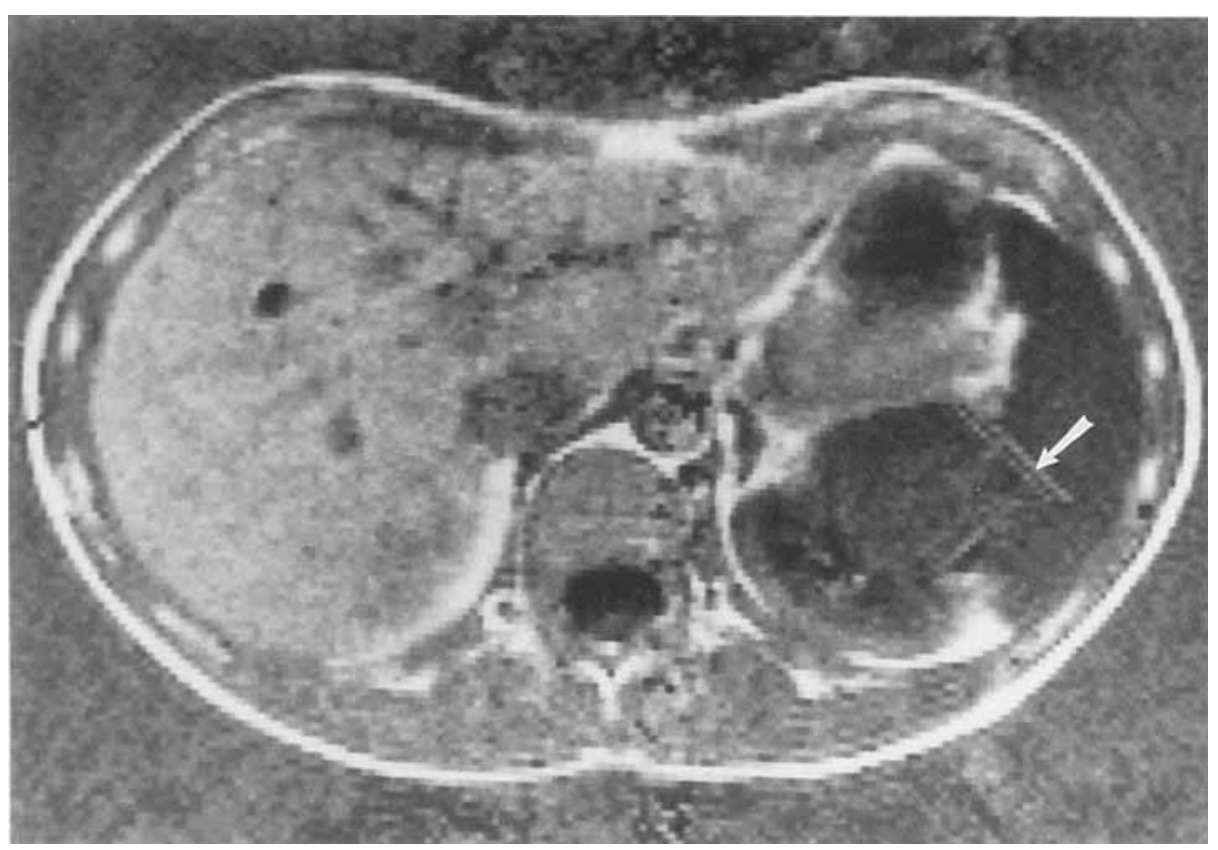

FIG. 6. Axial section through a normal liver, IR 1500/30/200. Phase correction was successful despite respiratory and flow artifact. One small area of phase correction error did occur (arrow).

head and knee) were easily phase-corrected and produced a good final result. Images of the liver sometimes required several attempts at phase correction using different thresholds for phase coherence. Good results were obtainable for most of the images, however. In particular, the liver images of one patient were severely degraded by respiratory artifact. Despite this degradation the algorithm was able to correct the phase of most of the images. Most of the difficulty occurred with the more cranial images which contained cardiac motion artifact and large areas of signal void in the lungs. Correction errors also occurred in part of images near the fringes of the radiofrequency coil.

\section{DISCUSSION}

Because of the nature of IR images, phase errors in the data will seriously degrade the images or render them useless. Phantom data can be used to phase correct the raw data, but this has several disadvantages. The field of view is limited by the size of the phantom, one must collect new phantom data if characteristics of the scanner change, and the technique cannot correct for magnetic susceptibility effects.

The regional phase correction algorithm described above does not suffer from these problems. The main disadvantage is that it is somewhat user dependent, in that thresholds for magnitude and phase coherence must be operator selected. Also the algorithm cannot determine reliably whether a particular area is short $\mathrm{T} 1$ or long $\mathrm{T} 1$, so the operator must review each image and decide whether the phase of the entire image must be shifted $180^{\circ}$. The time of operator interaction is small, and can be 
performed by an MRI technologist once he or she is familiarized with the process. Once thresholds are selected, image processing may proceed with no further intervention. The length of time required for phase correction was approximately $20-30 \mathrm{~min}$ for a $256 \times 256$ pixel image. Presumably, this time could be considerably shortened using an array processor.

Because of the difficulty encountered in phase correcting images near the diaphragm, it is anticipated that this technique will not work well with images of the chest because of cardiac and respiratory artifact. However, this speculation has not been explored, and it is conceivable that useful results may be obtainable using a gated pulse sequence.

Postprocessing of water-fat MR images involves correction of similar phase errors, and phase unwrapping has been applied to this situation as well (5). The algorithm described in this paper has also been applied to phase correction of water-fat images (6), with good results. Because of the larger number of cycles of phase errors in waterfat images, the algorithm is more prone to errors. This is compensated for by initially correcting phase with a uniform phantom and using the algorithm to correct only the phase errors which remain.

Several studies have demonstrated the usefulness of IR images (7-13). The advantage of phase-sensitive images over modulus images has also been demonstrated ( 3 , $4,14)$. While not all scanners will require significant phase correction, those which do will not be able to produce proper IR images without a means of correcting the phase. It is concluded that this regional phase correction technique is an effective way to obtain good IR images from a scanner with a significant amount of residual phase error.

\section{REFERENCES}

1. I. R. Young, D. R. Bailes, AND G. M. Bydder, Magn. Reson. Med. 2, 81 (1985).

2. C. J. G. BAKkeR, C. N. DE GRAaF, AND P. VAN DiJk, IEEE Trans. on Med. Imaging, MI-3, 197 (1984).

3. H. W. Park, M. H. Cho, ANd Z. H. Cho, Magn. Reson. Med. 3, 15 ( 1986 ).

4. P. R. Moran, N. G. Kumar, N. Karstaedt, and S. C. JaCkels, Magn. Reson. Imaging 4, 229 (1986).

5. H. N. YeUNG AND D. W. Kormos, Radiology 159, 783 (1986).

6. J. A. Borrello, T. L. Chenevert, C. R. Meyer, A. M. Aisen, And G. M. Glazer, Radiology 164, 531 ( 1987$)$.

7. W. Huk, W. Heindel, M. Deimling, And E. Stetter, J. Comput. Assist. Tomogr. 7, 468 (1983).

8. G. M. Bydder and I. R. YounG, J. Comput. Assist. Tomogr. 9, 659 (1985).

9. S. J. El Yousef, D. M. O'Connell, R. H. Duchesneau, M. J. Smith, C. A. Hubay, and S. P. GuYTON, Amer. J. Roentgenol. 145, 1 (1985).

10. D. D. STARK, J. WitTEnBERG, R. J. ButCh, AND J. T. FerruCCI, Radiology 165, 399 ( 1987 ).

11. T. C. Larson, O. W. Houser, and E. R. Laws, Mayo Clin. Proc. 62, 886 (1987).

12. M. V. Kulkarni, K. F. Lee, C. B. McArdle, J. W. Yeakley, and F. L. HaAr, Amer. J. Neuroradiol. 9, 5(1988).

13. D. A. Robinson, R. E. Steiner, And I. R. YounG, I. Comput. Assist. Tomogr. 12, 275 ( 1988 ).

14. R. T. Droege and S. M. AdamCZaK, Magn. Reson. Med. 3, 126 (1986). 